Stud. Univ. Babeş-Bolyai Math. 62(2017), No. 2, 151-162

DOI: $10.24193 /$ subbmath.2017.2.02

\title{
The first Zolotarev case in the Erdös-Szegö solution to a Markov-type extremal problem of Schur
}

\author{
Heinz-Joachim Rack
}

\begin{abstract}
Schur's [14] Markov-type extremal problem asks to find the maximum (1) $\sup _{-1 \leq \xi \leq 1} \sup _{P_{n} \in \mathbf{B}_{n, \xi, 2}}\left|P_{n}^{(1)}(\xi)\right|$, where $\mathbf{B}_{n, \xi, 2}=\left\{P_{n} \in \mathbf{B}_{n}: P_{n}^{(2)}(\xi)=0\right\} \subset \mathbf{B}_{n}=$ $\left\{P_{n}:\left|P_{n}(x)\right| \leq 1\right.$ for $\left.|x| \leq 1\right\}$ and $P_{n}$ is an algebraic polynomial of degree $\leq n$. Erdös and Szegö [3] found that for $n \geq 4$ this maximum is attained if $\xi= \pm 1$ and $P_{n} \in \mathbf{B}_{n, \xi, 2}$ is a (unspecified) member of the 1-parameter family of hard-core Zolotarev polynomials $Z_{n, t}$. Our first result centers around the proof in [3] for the initial case $n=4$ and is three-fold: $(i)$ the numerical value for (1) in ([3], (7.9)) is not correct, but sufficiently precise; (ii) from preliminary work in [3] can in fact be deduced a closed analytic expression for (1) if $n=4$, allowing numerical evaluation to any precision; (iii) even the explicit power form representation of an extremal $Z_{4, t}=Z_{4, t^{*}}$ can be deduced from [3], thus providing an exemplification of Schur's problem that seems to be novel. Additionally, we cross-check its validity twice: firstly by deriving $Z_{4, t^{*}}$ conversely from a general formula for $Z_{4, t}$ that we have given in [12], and secondly by applying Theorem 5.10 in [11]. We then turn to a generalized solution of Schur's problem, to $k$-th derivatives, by Shadrin [16]. Again we provide in explicit form the corresponding maximum as well as an extremizer polynomial for the first non-trivial degree $n=4$. Finally, we contribute to the fuller description of $Z_{4, t}$ by providing its critical points in explicit form.
\end{abstract}

Mathematics Subject Classification (2010): 26C05, 26D10, 41A10, 41A17, 41A29, 41A44, 41A50.

Keywords: Chebyshev, derivative, Erdös, extremal problem, inequality, Markov, polynomial, quartic, Schur, Shadrin, Szegö, Zolotarev. 


\section{Introduction}

The famous A. A. Markov inequality of 1889 [8] asserts an estimate on the size of the first derivative of an algebraic polynomial $P_{n}$ of degree $\leq n$ and can be restated as follows:

$$
\sup _{\xi \in \mathbf{I}} \sup _{P_{n} \in \mathbf{B}_{n}}\left|P_{n}^{(1)}(\xi)\right|=n^{2}=T_{n}^{(1)}(1)
$$

where $\mathbf{I}=[-1,1]$ and $\mathbf{B}_{n}=\left\{P_{n}:\left|P_{n}(x)\right| \leq 1\right.$ for $\left.x \in \mathbf{I}\right\}$. As indicated, this maximum will be attained if, up to the sign, $P_{n}=T_{n} \in \mathbf{B}_{n}$ is the $n$-th Chebyshev polynomial of the first kind on $\mathbf{I}$ (defined by $T_{n}(x)=2 x T_{n-1}(x)-T_{n-2}(x)$ with $T_{1}(x)=x$, $\left.T_{0}(x)=1\right)$ and if $\xi= \pm 1$, see e.g. ([10], p. 529), ([13], p. 123).

In 1919 I. Schur ([14], §2), inspired by (1.1), was led to the problem of finding the maximum of $\left|P_{n}^{(1)}(\xi)\right|$ under the additional restriction $P_{n}^{(2)}(\xi)=0$ : Determine $P_{n}=P_{n}^{*}$ which attains, for $n \geq 3$,

$$
\sup _{\xi \in \mathbf{I}} \sup _{P_{n} \in \mathbf{B}_{n, \xi, 2}}\left|P_{n}^{(1)}(\xi)\right|=n^{2} M_{n},
$$

where $\mathbf{B}_{n, \xi, 2}=\left\{P_{n} \in \mathbf{B}_{n}: P_{n}^{(2)}(\xi)=0\right\}$ and $M_{n}$ is a constant (depending on $n$ ). Schur $([14],(9))$ proved that there holds

$$
\sup _{\xi \in \mathbf{I}} \sup _{P_{n} \in \mathbf{B}_{n, \xi, 2}}\left|P_{n}^{(1)}(\xi)\right|<\frac{1}{2} n^{2}, \text { so that } M_{n}<\frac{1}{2} .
$$

In 1942 P. Erdös and G. Szegö addressed this problem of Schur and they provided the following solution ([3], Theorem 2):

The maximum (1.2) will be attained, for $n \geq 4$, only if $\xi=1$ and $P_{n}=P_{n}^{*}$ is a (unspecified) member of the 1-parameter family (with parameter $t$ ) of hard-core Zolotarev polynomials $\pm Z_{n, t}$; or if $\xi=-1$ and $P_{n}=P_{n}^{*}$ is a (unspecified) member of the family $\pm Z_{n, t}^{-}$, where $Z_{n, t}^{-}(x)=Z_{n, t}(-x)$.

We leave aside the simple case $n=3$ (with solution $\xi=0$ and $P_{3}=P_{3}^{*}= \pm T_{3}$ $([3]$, p. 466)). Henceforth we will confine ourselves to specify only one extremal polynomial $P_{n}^{*}$ for a given problem on $\mathbf{I}$, but will keep in mind that $-P_{n}^{*}$ as well as $\pm Q_{n}^{*}$, where $\pm Q_{n}^{*}(x)= \pm P_{n}^{*}(-x)$, may likewise be extremal. The solutions to (1.1) and (1.2) have in common that the maximum is attained at the endpoints $\xi= \pm 1$ of the unit interval $\mathbf{I}$. But, on the other hand, the solutions differ greatly when it comes to exhibit an explicit extremal polynomial from $\mathbf{B}_{n}$ resp. $\mathbf{B}_{n, \xi, 2}$ : Whereas in (1.1) an extremizer is, for all $n \geq 1$, the well-known $n$-th Chebyshev polynomial $T_{n}$ [13], the explicit power form of the intricate extremizers $Z_{n, t}$ in (1.2) remained arcane for all $n \geq 4$. This is due to the fact that for a general degree $n$ the explicit power form of a hard-core Zolotarev polynomial $Z_{n, t}$ is not known ([16], p. 1185). Rather, $Z_{n, t}$ can be expressed with the aid of elliptic functions (see ([1], pp. 280), ([10], p. 407), [18]) which amounts to an extremely complicated concoction of elliptic quantities ([17], p. $52)$.

It is a purpose of this note to provide, nearly one hundred years after the origin of 
Schur's problem, the explicit power form of a particular hard-core Zolotarev polynomial $Z_{n, t}=Z_{n, t^{*}}$ which is extremal for (1.2), at least for the first nontrivial case $n=4$. Such a solution was coined Schur polynomial in ([11], Section 5d), where a numerical method (solution of a system of nonlinear equations) is advised in order to determine it.

We will first tackle the explicit analytic expression for (1.2) if $n=4$. Once it has been established, to calculate its numerical value to arbitrary precision becomes immediate. Incidentally, we notice that the numerical value for $16 M_{4}$ as given in $([3],(7.9))$ is not correct from the third decimal place on. We then deduce, in three alternative fashions, an extremal hard-core Zolotarev polynomial $P_{4}^{*}=Z_{4, t^{*}}$ with optimal value $t^{*}$ of the parameter $t$. This Schur polynomial $P_{4}^{*}$ may well serve as illustrative example of the result in ([3], Theorem 2). Finally, we will consider a recent generalization of Schur's problem (1.2), due to A. Shadrin [16], to higher derivatives of $P_{n}$, and again we will exemplify the quartic case $n=4$. In a closing remark we reveal the critical points of $Z_{4, t}$ to get a fuller picture of the quartic hard-core Zolotarev polynomial.

\section{Analytical and numerical value of the maximum in the quartic case}

To determine the value in (1.2) for $n=4$ we rely on preliminary work in ([3], Section 7) and will therefore retain, for the reader's convenience, the notation used there. A sought-for extremal hard-core Zolotarev polynomial $P_{4}^{*}$ which solves (1.2) can be assumed to be from class $\mathbf{B}_{4,1,2}$ and be represented as, see ([3], (7.3)),

$$
P_{4}^{*}(x)=1-\lambda(1-x)\left(B_{4}-x\right)\left(y_{1}-x\right)^{2},
$$

where $\lambda, B_{4}, y_{1}$ are parameters which reflect properties of $P_{4}^{*}$, such as:

$$
P_{4}^{*}(-1)=-1, P_{4}^{*}\left(y_{1}\right)=1, P_{4}^{*(1)}\left(y_{1}\right)=0, P_{4}^{*}(1)=P_{4}^{*}\left(B_{4}\right)=1 .
$$

The first and second derivative of $P_{4}^{*}$ at $x=1$ read:

$$
P_{4}^{*(1)}(1)=\lambda\left(B_{4}-1\right)\left(1-y_{1}\right)^{2} \text { and } P_{4}^{*(2)}(1)=2 \lambda\left(y_{1}-1\right)\left(2\left(1-B_{4}\right)-\left(y_{1}-1\right)\right),
$$

so that the condition $P_{4}^{*(2)}(1)=0$ yields $y_{1}=3-2 B_{4}$ which, when inserted into $P_{4}^{*(1)}(1)$, eliminates there the parameter $y_{1}$. From $P_{4}^{*}(-1)=-1$ one deduces, upon inserting the said value of $y_{1}$, that

$$
\lambda=\frac{1}{\left(B_{4}+1\right)\left(4-2 B_{4}\right)^{2}},
$$

see (2.1). This implies

The identity

$$
P_{4}^{*(1)}(1)=\frac{\left(B_{4}-1\right)^{3}}{\left(B_{4}-2\right)^{2}\left(B_{4}+1\right)} .
$$

$$
\frac{2}{B_{4}-1}=\frac{11-\sqrt{33}+2 \sqrt{5(5+\sqrt{33})}}{8},
$$

which is given in an equivalent form in $\left([3],(7.8)\right.$ ), allows to evaluate $B_{4}$ (see (3.2) below). Inserting this value of $B_{4}$ into the preceding expression for $P_{4}^{*(1)}(1)$ eventually 
yields the analytical expression for the maximum, which can be evaluated numerically to any desired precision:

$$
\begin{aligned}
P_{4}^{*(1)}(1) & =\sup _{\xi \in \mathbf{I}} \sup _{P_{4} \in \mathbf{B}_{4, \xi, 2}}\left|P_{4}^{(1)}(\xi)\right|=16 M_{4} \\
& =\frac{-561+161 \sqrt{33}+\sqrt{30(15215+3329 \sqrt{33})}}{288} \\
& =4.7876468942 \ldots,
\end{aligned}
$$

being a root of $P_{4}(x)=-65536-39424 x-1915 x^{2}+1683 x^{3}+216 x^{4}$.

By contrast, Formula (7.9) in [3] states that

$$
P_{4}^{*(1)}(1)=4.7881 \ldots
$$

holds, a value which is now seen to be biased in the third and fourth decimal place. But that bias does not harm the argument in [3] for $n=4$ since the first two valid decimal places are sufficiently conclusive for $P_{4}^{*}$ to be the extremal element (as a comparison is drawn with competitor polynomial $T_{4}$ and value $\left|T_{4}^{(1)}\left(\frac{1}{\sqrt{6}}\right)\right|=4.3546 \ldots$, see $([3],(7.2)))$.

The constant $M_{4}$ itself can thus be represented as

$$
\begin{aligned}
M_{4} & =\frac{P_{4}^{*(1)}(1)}{16}=\sup _{\xi \in \mathbf{I}} \sup _{P_{4} \in \mathbf{B}_{4, \xi, 2}} \frac{\left|P_{4}^{(1)}(\xi)\right|}{4^{2}} \\
& =\frac{-561+161 \sqrt{33}+\sqrt{30(15215+3329 \sqrt{33})}}{4608} \\
& =0.2992279308 \ldots
\end{aligned}
$$

We note that according to $([3],(1.3),(1.4))$ there holds $\lim _{n \rightarrow \infty} M_{n}=0.3124 \ldots$. Schur ([14], p. 277) had obtained the weaker result $0.217 \ldots \leq \lim \sup _{n \rightarrow \infty} M_{n} \leq 0.465 \ldots$.

\section{Explicit power form representation of an extremal hard-core Zolotarev polynomial in the quartic case}

Having expressed the parameters $\lambda=\lambda\left(B_{4}\right)$ and $y_{1}=y_{1}\left(B_{4}\right)$ as functions of $B_{4}$ alone and knowing the value of the constant $B_{4}$, it is possible to even retrieve the explicit power form of an extremal $P_{4}^{*}$. In fact, according to the preceding Section we have

$$
\begin{aligned}
P_{4}^{*}(x) & =1-\lambda(1-x)\left(B_{4}-x\right)\left(y_{1}-x\right)^{2} \\
& =1-\frac{(1-x)\left(B_{4}-x\right)\left(3-2 B_{4}-x\right)^{2}}{\left(B_{4}+1\right)\left(4-2 B_{4}\right)^{2}}
\end{aligned}
$$


Inserting now

$$
\begin{aligned}
B_{4} & =\frac{177-17 \sqrt{33}+\sqrt{30(527+97 \sqrt{33})}}{144} \\
& =1.8034303689 \ldots
\end{aligned}
$$

and expanding (3.1) leads us, after some algebraic manipulations, to the explicit power form representation of an extremal quartic hard-core Zolotarev polynomial $P_{4}^{*}$ with

$$
P_{4}^{*}(x)=\sum_{i=0}^{4} a_{i}^{*} x^{i}
$$

and with coefficients

$$
\begin{aligned}
& a_{0}^{*}=\frac{21297-2081 \sqrt{33}-\sqrt{30(3160847+628577 \sqrt{33})}}{9216}=-0.5328330303 \ldots \\
& a_{1}^{*}=\frac{291-1139 \sqrt{33}-\sqrt{30(-1236313+427337 \sqrt{33})}}{4608}=-2.6688925571 \ldots \\
& a_{2}^{*}=\frac{-849+161 \sqrt{33}+\sqrt{30(15215+3329 \sqrt{33})}}{384}=2.8407351706 \ldots \\
& a_{3}^{*}=\frac{4317+1139 \sqrt{33}+\sqrt{30(-1236313+427337 \sqrt{33})}}{4608}=3.6688925571 \ldots \\
& a_{4}^{*}=\frac{-921-1783 \sqrt{33}-\sqrt{330(-59555+64243 \sqrt{33})}}{9216}=-2.3079021403 \ldots
\end{aligned}
$$

These optimal coefficients $a_{i}^{*}$ are roots of the following respective quartic polynomials $P_{4, i}$ with integer coefficients:

$$
\begin{aligned}
& P_{4,0}(x)=-7951932-7463259 x+11697424 x^{2}-4089024 x^{3}+442368 x^{4} \\
& P_{4,1}(x)=12221+273251 x-7120 x^{2}-3492 x^{3}+13824 x^{4} \\
& P_{4,2}(x)=-236196-112023 x+17720 x^{2}+13584 x^{3}+1536 x^{4} \\
& P_{4,3}(x)=288684-303831 x+65348 x^{2}-51804 x^{3}+13824 x^{4} \\
& P_{4,4}(x)=314928+2644083 x-861584 x^{2}+176832 x^{3}+442368 x^{4} .
\end{aligned}
$$

This result constitutes, to the best of our knowledge, the first explicit example of an extremal $P_{n}^{*}$ which solves Schur's problem according to Erdös-Szegö ([3], Theorem 2) (here for the first Zolotarev case $n=4$ ). It is therefore worth summarizing the properties of that Schur polynomial $P_{4}^{*} \in \mathbf{B}_{4}$ : 
(i) The equiripple property on I, i.e., 4 alternation points, including the endpoints \pm 1 :

$$
\begin{aligned}
P_{4}^{*}(-1) & =-1 \\
P_{4}^{*}\left(y_{1}\right) & =1 \text { and } P_{4}^{*(1)}\left(y_{1}\right)=0, \text { where } \\
y_{1} & =\frac{1}{72}(39+17 \sqrt{33}-\sqrt{30(527+97 \sqrt{33})})=-0.6068607378 \ldots, \\
P_{4}^{*}\left(y_{2}\right) & =-1 \text { and } P_{4}^{*(1)}\left(y_{2}\right)=0, \text { where } \\
y_{2} & =\frac{1}{72}(105-\sqrt{33}-\sqrt{30(95+17 \sqrt{33})})=0.3226516930 \ldots, \\
P_{4}^{*}(1) & =1 .
\end{aligned}
$$

(ii) The Zolotarev property at three points $A_{4}<B_{4}<C_{4}$ to the right of $\mathbf{I}$ (of which $B_{4}$ and $C_{4}$ are two additional alternation points)

$$
\begin{aligned}
P_{4}^{*(1)}\left(A_{4}\right) & =0, \text { where } \\
A_{4} & =\frac{279+25 \sqrt{33}+\sqrt{30(2879+561 \sqrt{33})}}{576}=1.4764907146 \ldots, \\
P_{4}^{*}\left(B_{4}\right) & =1, \text { where } B_{4} \text { is given in }(3.2), \\
P_{4}^{*}\left(C_{4}\right) & =-1, \text { where } \\
C_{4} & =\frac{201+55 \sqrt{33}-\sqrt{330(61+19 \sqrt{33})}}{144}=1.9444055070 \ldots
\end{aligned}
$$

Additionally, by construction, $P_{4}^{*}$ satisfies

$$
\begin{aligned}
& P_{4}^{*(2)}(1)=2\left(a_{2}^{*}+3 a_{3}^{*}+6 a_{4}^{*}\right)=0, \text { i.e., } P_{4}^{*} \in \mathbf{B}_{4,1,2} \\
& P_{4}^{*(1)}(1)=a_{1}^{*}+2 a_{2}^{*}+3 a_{3}^{*}+4 a_{4}^{*}=16 M_{4}, \text { see }(2.3),
\end{aligned}
$$

and we add, by inspection, that

$$
a_{3}^{*}=1-a_{1}^{*} \text { and } a_{4}^{*}=-a_{0}^{*}-a_{2}^{*} .
$$

That particular hard-core Zolotarev polynomial $P_{4}^{*}$ may well serve as elucidating example to provide for explanation purposes in lectures or expository writings on Schur's problem, respectively on its solution by Erdös-Szegö, see e.g. [4].

\section{Alternative deductions of an explicit extremal hard-core Zolotarev polynomial in the quartic case}

In ([12], p. 357) we have provided explicit expressions for the parameterized coefficients of an arbitrary fourth-degree hard-core Zolotarev polynomial on $\mathbf{I}$. But since the assumption was made there that it attains the value 1 at $x=-1$, we prefer to consider here the negative form of that polynomial in order to be compliant with [3]. We hence set

$$
Z_{4, t}(x)=\sum_{i=0}^{4}-a_{i}(t) x^{i}, \text { with } 1<t<1+\sqrt{2}
$$


where the coefficients $a_{i}(t)$ read as follows:

$$
\begin{aligned}
a_{0}(t)= & \left(-a^{5}-b^{3}+a^{4}(-2+3 b)+a^{3}\left(-1+6 b-3 b^{2}\right)+\right. \\
& \left.+a\left(3 b^{2}-2 b^{3}\right)+a^{2}\left(3 b+2 b^{2}+b^{3}\right)\right) \kappa, \\
a_{1}(t)= & \left(a^{2}\left(-16 b+8 b^{2}\right)+a\left(-12 b+8 b^{2}-4 b^{3}\right)\right) \kappa, \\
a_{2}(t)= & \left(a^{2}(8-16 b)+6 b-4 b^{2}+2 b^{3}+a\left(6-4 b+2 b^{2}\right)\right) \kappa, \\
a_{3}(t)= & \left(-4+8 a^{2}+8 b+8 a b-4 b^{2}\right) \kappa, \\
a_{4}(t)= & (-4-6 a+2 b) \kappa
\end{aligned}
$$

with

$$
\begin{aligned}
\kappa & =\frac{1}{(1+a)^{2}(-a+b)^{3}} \\
a & =\frac{1-3 t-t^{2}-t^{3}}{(1+t)^{3}} \\
b & =\frac{1+t+3 t^{2}-t^{3}}{(1+t)^{3}} .
\end{aligned}
$$

Here $a$ and $b$ with $a<b$ are the alternation points of $Z_{4, t}$ in the interior of $\mathbf{I}$. We now proceed to determine the optimal parameter $t=t^{*}$ and the corresponding explicit coefficients $-a_{i}\left(t^{*}\right)$ of an extremal polynomial $Z_{4, t^{*}}$ with $Z_{4, t^{*}}(x)=\sum_{i=0}^{4}-a_{i}\left(t^{*}\right) x^{i}$ which, according to the general result in ([3], Theorem 2), solves Schur's problem (1.2) for $n=4$.

The assumption $Z_{4, t} \in \mathbf{B}_{4,1,2}$, i.e., $Z_{4, t}^{(2)}(1)=0$, implies

$$
a_{2}(t)+3 a_{3}(t)+6 a_{4}(t)=0 \text {. }
$$

Employing the definition of $a_{i}(t)$ in $(4.2),(4.3)$ this amounts to the following equation, after some algebraic manipulations:

$$
\frac{(1+t)^{3}(3+t(2+t))(-2+t(-7+t(1+3(-1+t) t)))}{4\left(t+t^{3}\right)^{2}}=0 .
$$

The numerator vanishes, for $1<t<1+\sqrt{2}$, only if we choose

$$
t=t^{*}=\frac{3+\sqrt{33}+\sqrt{30(-1+\sqrt{33})}}{12}=1.7229220588 \ldots,
$$

which is a root of the polynomial $P_{4}(x)=-2-7 x+x^{2}-3 x^{3}+3 x^{4}$. Inserting the optimal parameter (4.6) into the coefficients $-a_{i}(t)$ of $Z_{4, t}$, see (4.2), (4.3), shows that $-a_{i}\left(t^{*}\right)$ indeed coincides for $i=0,1,2,3,4$ with $a_{i}^{*}$ as given in (3.3). We check only the coefficient $-a_{4}(t)$ and leave it to the reader to check the remaining coefficients:

$$
-a_{4}(t)=\frac{4+6 a-2 b}{(1+a)^{2}(-a+b)^{3}}=\frac{(1-t)(1+t)^{9}}{32 t^{3}\left(1+t^{2}\right)^{2}},
$$

and inserting now $t=t^{*}$ according to (4.6) indeed yields $-a_{4}\left(t^{*}\right)=a_{4}^{*}$ as given in (3.3). After all, we so obtain an alternative and independent deduction of the extremal hard-core Zolotarev polynomial $P_{4}^{*}=Z_{4, t^{*}}$ which we had already found in Section 3 , based on preliminary work in [3]. 
A third argument can be brought forward to prove that $P_{4}^{*}=Z_{4, t^{*}}$ is a soughtfor extremizer in (1.2) for $n=4$, see ([11], Theorem 5.10): It suffices to verify that the following five equations hold true

$$
\begin{array}{r}
-1+2\left(-y_{1}+y_{2}\right)-\left(1+B_{4}-C_{4}\right)=0 \\
1+2\left(-y_{1}^{2}+y_{2}^{2}\right)-\left(1+B_{4}^{2}-C_{4}^{2}\right)=0 \\
-1+2\left(-y_{1}^{3}+y_{2}^{3}\right)-\left(1+B_{4}^{3}-C_{4}^{3}\right)=0 \\
\frac{16\left(A_{4}-1\right)^{2}}{\left(B_{4}-1\right)\left(C_{4}-1\right)}=1+2\left(\frac{2}{A_{4}-1}-\frac{1}{B_{4}-1}-\frac{1}{C_{4}-1}\right) \\
A_{4}=\frac{3}{8}\left(B_{4}+C_{4}\right)-\frac{1}{4}\left(y_{1}+y_{2}\right),
\end{array}
$$

where $y_{1}$ and $y_{2}$ are defined in (3.5), $A_{4}$ and $C_{4}$ are defined in (3.6), and $B_{4}$ is defined in (3.2). We leave it to the reader to check the validity of equations (4.8) - (4.12). Summarizing, we have thus established

Proposition 4.1. Polynomial $P_{4}^{*}$ with $P_{4}^{*}(x)=\sum_{i=0}^{4} a_{i}^{*} x^{i}$ and explicit coefficients $a_{i}^{*}$ $(i=0,1,2,3,4)$ according to (3.3) is a sought-for extremal hard-core Zolotarev polynomial of degree four which solves, according to Erdös-Szegö ([3], Theorem 2), Schur's problem (1.2) for $n=4$. The corresponding maximum

$$
\sup _{\xi \in \mathbf{I}} \sup _{P_{4} \in \mathbf{B}_{4, \xi, 2}}\left|P_{4}^{(1)}(\xi)\right|=16 M_{4}
$$

is explicitly given in (2.3), so that $M_{4}$ equals the constant given in (2.5).

\section{A generalized Schur problem and its solution for the quartic case}

A. A. Markov's inequality (1.1) for the first derivative of $P_{n}$ was generalized in 1892 by his half-brother V. A. Markov ([9], p. 93) to the $k$-th derivative and can be restated as follows, see also ([10], p. 545), ([13], Theorem 2.24):

$$
\sup _{\xi \in \mathbf{I}} \sup _{P_{n} \in \mathbf{B}_{n}}\left|P_{n}^{(k)}(\xi)\right|=\prod_{j=0}^{k-1} \frac{n^{2}-j^{2}}{2 j+1}=T_{n}^{(k)}(1),(1 \leq k \leq n),
$$

indicating that the maximum is attained if $P_{n}=T_{n}$ and $\xi=1$. Shadrin [16] has analogously generalized Schur's problem (1.2) to the $k$-th derivative. This generalized problem can be stated as follows:

Determine, for $1 \leq k \leq n-2$ and $n \geq 4$, an algebraic polynomial $P_{n}=P_{n}^{*}$ of degree $\leq n$ which attains the maximum

$$
\sup _{\xi \in \mathbf{I}} \sup _{P_{n} \in \mathbf{B}_{n, \xi, k+1}}\left|P_{n}^{(k)}(\xi)\right|=\prod_{j=0}^{k-1} \frac{n^{2}-j^{2}}{2 j+1} M_{n, k}=T_{n}^{(k)}(1) M_{n, k},
$$

where $\mathbf{B}_{n, \xi, k+1}=\left\{P_{n} \in \mathbf{B}_{n}: P_{n}^{(k+1)}(\xi)=0\right\}$ and $M_{n, k}$ is a constant (depending on $n$ and $k$ ). Shadrin ([16], Proposition 4.4) found that, for $k \geq 2$, this maximum is attained 
if $\xi=1$ and $P_{n}=P_{n}^{*} \in \mathbf{B}_{n, 1, k+1}$ is a Zolotarev polynomial $Z_{n}$ (not necessarily a hardcore one), or if $\xi=\omega_{k, n}$, the rightmost zero of $T_{n}^{(k+1)}$, and $P_{n}=P_{n}^{*}=T_{n}$, so that altogether there holds:

$$
\sup _{\xi \in \mathbf{I}} \sup _{P_{n} \in \mathbf{B}_{n, \xi, k+1}}\left|P_{n}^{(k)}(\xi)\right|=\max \left\{\left|Z_{n}^{(k)}(1)\right|,\left|T_{n}^{(k)}\left(\omega_{k, n}\right)\right|\right\} .
$$

We are now going to determine that maximum as well as an extremizer polynomial for the quartic case $n=4$ and for the second derivative, i.e., $k=2=n-2$ (the case $k=1$ is settled in Proposition 4.1). It is well known that Zolotarev polynomials $Z_{n}$ of degree $n \geq 4$ on $\mathbf{I}$ satisfy $\left\|Z_{n}\right\|_{\infty}=1$ (maximum-norm) and exhibit at least $n$ equiripple points on $\mathbf{I}$ where the values \pm 1 are attained alternately, see ([16], p. 1190). Apart from sign and reflection, the Zolotarev polynomial $Z_{4}$ takes on the role (see also ([1], pp. 280), ([10], p. 406)):

(i) $Z_{4}=T_{3}$, with $T_{3}(x)=-3 x+4 x^{3}$,

(ii) $Z_{4}=T_{4}$, with $T_{4}(x)=1-8 x^{2}+8 x^{4}$,

(iii) $Z_{4}=T_{4, \beta}$, with $T_{4, \beta}(x)=T_{4}\left(\frac{2 x-\beta+1}{1+\beta}\right)$

where $1<\beta \leq 1+2 \tan ^{2}\left(\frac{\pi}{8}\right)=7-4 \sqrt{2}=1.3431457505 \ldots$,

(iv) $Z_{4}=Z_{4, t}$, the hard-core Zolotarev polynomial, as given in (4.1).

We first calculate $\left|Z_{4}^{(2)}(1)\right|$, subject to the constraint $Z_{4}^{(3)}(1)=0$, and observe that polynomials (i), (ii), (iii) cannot be extremal due to $T_{3}^{(3)}(1)=24 \neq 0$, resp. $T_{4}^{(3)}(1)=$ $192 \neq 0$, resp. $T_{4, \beta}^{(3)}(1)=\frac{1536(3-\beta)}{(1+\beta)^{4}} \neq 0$ if $1<\beta \leq 7-4 \sqrt{2}$. For polynomial (iv) we get, after some algebraic manipulations,

$$
\left|Z_{4, t}^{(3)}(1)\right|=\left|\frac{3(1+t)^{6}\left(-1+t\left(-8+2 t+3 t^{3}\right)\right)}{8 t^{3}\left(1+t^{2}\right)^{2}}\right| .
$$

The numerator vanishes for $1<t<1+\sqrt{2}$ only if

$$
t=t^{* *}=\frac{1+\sqrt{2(-1+\sqrt{3})}}{\sqrt{3}}=1.2759444802 \ldots
$$

Inserting this parameter $t^{* *}$ into $\left|Z_{4, t}^{(2)}(1)\right|$ yields, again after some manipulations,

$$
\left|Z_{4, t^{* *}}^{(2)}(1)\right|=\left|-12-\frac{22}{\sqrt{3}}+4 \sqrt{\frac{10}{3}+2 \sqrt{3}}\right|=14.2729495641 \ldots .
$$

In view of (5.3), we have next to compare (5.6) to $\left|T_{4}^{(2)}\left(\omega_{2,4}\right)\right|$. Since the only, and hence the rightmost, zero of $T_{4}^{(3)}$ is $\omega_{2,4}=0$, we get

$$
\left|T_{4}^{(2)}(0)\right|=|-16|=16>\left|Z_{4, t^{* *}}^{(2)}(1)\right| \text {. }
$$


So eventually we arrive at the identity

$$
\begin{aligned}
\sup _{\xi \in \mathbf{I}} \sup _{P_{4} \in \mathbf{B}_{4, \xi, 3}}\left|P_{4}^{(2)}(\xi)\right| & =\max \left\{\left|Z_{4}^{(2)}(1)\right|,\left|T_{4}^{(2)}(0)\right|\right\}=16 \\
& =\prod_{j=0}^{1} \frac{4^{2}-j^{2}}{2 j+1} M_{4,2}=80 M_{4,2},
\end{aligned}
$$

yielding $M_{4,2}=\frac{1}{5}=0.2$. Summarizing, we have thus established

Proposition 5.1. Polynomial $P_{4}^{*}=T_{4}$ with $T_{4}(x)=1-8 x^{2}+8 x^{4}$ is a sought-for extremal polynomial of degree four which solves, according to Shadrin ([16], Proposition 4.4), the generalized Schur problem (5.2) for $n=4$ and $k=2$. The corresponding maximum $\sup _{\xi \in \mathbf{I}} \sup _{P_{4} \in \mathbf{B}_{4, \xi, 3}}\left|P_{4}^{(2)}(\xi)\right|=80 M_{4,2}$ is 16 , so that $M_{4,2}$ equals the constant $\frac{1}{5}$.

Shadrin ([16], Theorem 7.1) has added to (5.3) the following estimate which can be viewed as an extension, to the $k$-th derivative, of Schur's estimate (1.3):

$$
\sup _{\xi \in \mathbf{I}} \sup _{P_{n} \in \mathbf{B}_{n, \xi, k+1}}\left|P_{n}^{(k)}(\xi)\right| \leq \prod_{j=0}^{k-1} \frac{n^{2}-j^{2}}{2 j+1} \lambda_{n, k}=T_{n}^{(k)}(1) \lambda_{n, k} \quad(1 \leq k \leq n-2),
$$

where $\lambda_{n, k}=\frac{1}{k+1} \cdot \frac{n-1}{n-1+k}$.

Thus for $k=2$ and $n=4$ we get $\lambda_{4,2}=\frac{1}{3} \cdot \frac{3}{5}=\frac{1}{5}=0.2=M_{4,2}$, see (5.7). However, for $k=1$ and $n=4$ we get $\lambda_{4,1}=\frac{1}{2} \cdot \frac{3}{4}=\frac{3}{8}=0.375>M_{4}=0.299 \ldots$, see $(2.5)$ and ([16], Remark 5.5).

\section{Concluding remarks}

1. In deducing (2.3) we have been guided by a computer algebra system which the authors of [3], who have paved the way, certainly did not have at their disposal.

2. Our explicit power form representation ([12], p. 357) for the fourth hardcore Zolotarev polynomial $Z_{4, t}$ remained unnoticed, and several related formulas have been published afterwards, e.g. ([2], p. 184), ([15], p. 242), ([18], p. 721). Shadrin [15] attributes his formula (with a different range of the parameter $t$ ) to V. A. Markov [9] and remarks: But already for $n=4$ it seems that nobody really believed that an explicit form can be found. As a matter of fact it was, by V. Markov in 1892. In a private communication Professor Shadrin kindly called our attention to p. 73 in [9] from which his formula can be recovered. However, one has first to exploit the relation $4 z=t^{3}+t$ (see p. 71 in [9]), then fix the parameter $\alpha$ and finally rearrange the Taylor form of the given fourth-degree polynomial, centered at $x_{0}=2 z$, to the usual power form centered at $x_{0}=0$. It is under these side conditions that priority for the power form representation of $Z_{4, t}$ belongs indeed to V. A. Markov [9].

3. In Section 4 we have alternatively deduced the Schur polynomial $P_{4}^{*}$ from the explicit power form $Z_{4, t}(x)=\ldots$ as given, up to the sign, in ([12], p. 357). $P_{4}^{*}$ can 
likewise be deduced from the explicit power form $Z_{4}(x, t)=\ldots$ as given in ([15], p. 242 ), however instead of $Z_{4, t}^{(2)}(1)=0$ (see (4.4)) one has then to set $Z_{4}^{(2)}(-1, t)=0$.

4. For the quartic Schur polynomial $P_{4}^{*}=Z_{4, t^{*}}$ we have determined its five critical points $y_{1}, y_{2} \in \mathbf{I}$ and $A_{4}, B_{4}, C_{4}$ with $1<A_{4}<B_{4}<C_{4}$. As $Z_{4, t^{*}}$ is a special case of the general quartic hard-core Zolotarev polynomial $Z_{4, t}$ it is desirable to know the corresponding five (general) critical points of $Z_{4, t}$ as well. These are, as can be verified by insertion: $y_{1}(t)=a(t)=a$ and $y_{2}(t)=b(t)=b$ as given in (4.3), and furthermore

$$
\begin{aligned}
& A_{4}(t)=\frac{1+4 t+2 t^{2}+4 t^{3}+t^{4}}{2(-1+t)(1+t)^{3}} \\
& B_{4}(t)=\frac{1+2 t+6 t^{3}-t^{4}}{(-1+t)(1+t)^{3}} \\
& C_{4}(t)=\frac{-1+6 t+2 t^{3}+t^{4}}{(-1+t)(1+t)^{3}} .
\end{aligned}
$$

Choosing $t=t^{*}$ according to (4.6) takes us back to the five critical points of $P_{4}^{*}=Z_{4, t^{*}}$.

5. The optimal parameter $t=t^{*}$ according to (4.6) which selects the quartic Schur polynomial $Z_{4, t^{*}}$ among all $Z_{4, t}$ with $1<t<1+\sqrt{2}$ can alternatively be determined as follows: In (4.11) replace $A_{4}$ by $A_{4}(t), B_{4}$ by $B_{4}(t)$ and $C_{4}$ by $C_{4}(t)$ according to (6.1) - (6.3). Then solve this generalized equation (4.11) for the unknown number $t$. The solution will turn out as $t=t^{*}$.

6. As some progress has been achieved in the computation of $Z_{n, t}$ for the next higher polynomial degrees $n \geq 5$ (see [5], [6], [7], [11]), we hope that we will be able to extend our results to some $n \geq 5$. Meanwhile, we have succeeded so for the case $n=5$. (The second Zolotarev case in the Erdös-Szegö solution to a Markov-type extremal problem of Schur, J. Numer. Anal. Approx. Theory 46(2017), to appear).

\section{References}

[1] Achieser, N.I., Theory of approximation, Dover Publications, Mineola N.Y., 2003 (Russian 1947).

[2] Collins, G.E., Application of quantifier elimination to Solotareff's approximation problem, in Stability Theory: Hurwitz Centenary Conference, (R. Jeltsch et al., eds.), Ascona 1995, Birkhäuser Verlag, Basel, 1996 (ISNM 121), 181-190.

[3] Erdös, P., Szegö, G., On a problem of I. Schur, Ann. Math., 43(1942), 451-470.

[4] Finch, S.R., Zolotarev-Schur Constant, in Finch, S.R., Mathematical Constants, Cambridge University Press, Cambridge (UK), 2003, 229-231.

[5] Grasegger, G., Vo, N. Th., An algebraic-geometric method for computing Zolotarev polynomials, Technical Report no. 16-02, RISC Report Series, Johannes Kepler University, Linz, Austria, 2016, 1-17.

[6] Lazard, D., Solving Kaltofen's challenge on Zolotarev's approximation problem, in Proceedings of International Symposium on Symbolic and Algebraic Computation (ISSAC), (J.-G. Dumas, ed.), Genova 2006, ACM, New York, 2006, 196-203. 
[7] Malyshev, V.A., The Abel equation, St. Petersburg Math. J., 13(2002), 893-938 (Russian 2001).

[8] Markov, A.A., On a question of D.I. Mendeleev, Zapiski. Imper. Akad. Nauk., St. Petersburg, 62(1889), 1-24 (Russian).

[9] Markov, V.A., On functions deviating least from zero, Izdat. Akad. Nauk., St. Petersburg, 1892, iv + 111 (Russian).

[10] Milovanović, G.V., Mitrinović, D.S., Rassias, Th.M., Topics in polynomials: Extremal problems, inequalities, zeros, World Scientific, Singapore, 1994.

[11] Peherstorfer, F., Schiefermayr, K., Description of extremal polynomials on several intervals and their computation. II, Acta Math. Hungar., 83(1999), 59-83.

[12] Rack, H.-J., On polynomials with largest coefficient sums, J. Approx. Theory, 56(1989), 348-359.

[13] Rivlin, Th.J., Chebyshev polynomials, 2nd ed., Wiley, New York, 1990.

[14] Schur, I., Über das Maximum des absoluten Betrages eines Polynoms in einem gegebenen Intervall, Math. Z., 4(1919), 271-287.

[15] Shadrin, A., Twelve proofs of the Markov inequality, in Approximation Theory: A volume dedicated to Borislav Bojanov, (D.K. Dimitrov et al., eds.), M. Drinov Acad. Publ. House, Sofia, 2004, 233-298.

[16] Shadrin, A., The Landau-Kolmogorov inequality revisited, Discrete Contin. Dyn. Syst., 34(2014), 1183-1210.

[17] Todd, J., A legacy from E.I. Zolotarev (1847-1878), Math. Intell., 10(1988), 50-53.

[18] Vlček, M., Unbehauen, R., Zolotarev polynomials and optimal FIR filters, IEEE Trans. Signal Process., 47(1999), 717-730. Corrections: IEEE Trans. Signal Process., 48(2000), 2171.

Heinz-Joachim Rack

Dr. Rack Consulting GmbH

Steubenstrasse 26 a

58097 Hagen, Germany

e-mail: heinz-joachim.rack@drrack.com 